PROCEEDINGS OF THE

AMERICAN MATHEMATICAL SOCIETY

Volume 127, Number 1, January 1999, Pages 217-221

S 0002-9939(99)04690-0

\title{
A CONVOLUTION ESTIMATE FOR A MEASURE ON A CURVE IN $\mathbb{R}^{4}$. II
}

\author{
DANIEL M. OBERLIN
}

(Communicated by Christopher D. Sogge)

\begin{abstract}
This paper contains almost-sharp $L^{p}-L^{q}$ convolution estimates for measures on the curve $\left(t, t^{2}, t^{3}, t^{4}\right)$ in $\mathbb{R}^{4}$.
\end{abstract}

Let $\gamma$ be the curve in $\mathbb{R}^{4}$ defined by $\gamma(t)=\left(t, t^{2}, t^{3}, t^{4}\right), t \in \mathbb{R}$. For an interval $I \subseteq \mathbb{R}$ we consider the convolution operator $T$ on $\mathbb{R}^{4}$ defined by

$$
T f(x)=\int_{I} f(x-\gamma(t)) d t, \quad x \in \mathbb{R}^{4}
$$

Our interest is in determining the type-set $\mathcal{T}$ of $T$ - the set of all $\left(\frac{1}{p}, \frac{1}{q}\right) \in[0,1] \times[0,1]$ such that $T\left(L^{p}\left(\mathbb{R}^{4}\right)\right) \subseteq L^{q}\left(\mathbb{R}^{4}\right)$. Let $A=(0,0), B=\left(\frac{4}{10}, \frac{3}{10}\right), C=\left(\frac{5}{10}, \frac{4}{10}\right)$, $D=\left(\frac{6}{10}, \frac{5}{10}\right), E=\left(\frac{7}{10}, \frac{6}{10}\right)$, and $F=(1,1)$. Since $T$ is a convolution operator, $\mathcal{T}$ lies on or below the closed segment $[A, F]$. Estimating $T f$ when $f$ is the characteristic function of a small ball shows that $\mathcal{T}$ lies on or above the line through $A$ and $B$ and so, by duality, on or below the line through $F$ and $E$. Estimating $T f$ when $f$ is the characteristic function of

$$
[0, \varepsilon] \times\left[0, \varepsilon^{2}\right] \times\left[0, \varepsilon^{3}\right] \times\left[0, \varepsilon^{4}\right]
$$

shows that $\mathcal{T}$ lies on or above the line through $B$ and $E$. Thus $\mathcal{T}$ lies inside the closed quadrilateral $A B E F$ (the closed convex hull of $A, B, E$, and $F$ ). If the interval of integration $I$ is unbounded, then homogeneity considerations show that $\mathcal{T} \subseteq[B, E]$. It is conjectured that these necessary conditions for the boundedness of $\bar{T}$ are also sufficient. By duality and interpolation, it would suffice to show that $B \in \mathcal{T}$ if $I=(0, \infty)$. The best previous result $[\mathrm{O} 1]$ is that $[C, D] \subseteq \mathcal{T}$ for any $I$. Here we establish an almost sharp result when $I$ is bounded.

Theorem. If $I$ is bounded, then, excepting possibly the segments $(A, B],[B, C)$, $(D, E]$, and $[E, F)$, the closed quadrilateral $A B E F$ lies in $\mathcal{T}$.

Proof. Let $G=\left(\frac{1}{2}, \frac{1}{2}\right)$. By duality, interpolation, and the result of [O1], it is enough to show that $[G, B) \subseteq \mathcal{T}$. And a homogeneity argument shows that it is enough to do this for $I=[0,1]$. To this end we will study an analytic family $T_{z}$ of convolution operators given formally by

$$
T_{z} f(x)=\frac{1}{\Gamma\left(\frac{(z+1)}{2}\right)} \int_{0}^{1} \int_{-1}^{1} f\left(x-\gamma(t)-u \gamma^{\prime \prime}(t)\right)|u|^{z} d u d t .
$$

Received by the editors May 12, 1997.

1991 Mathematics Subject Classification. Primary 42B15.

The author was partially supported by a grant from the National Science Foundation. 
We will establish, with acceptable estimates on the growth of the operator norms, that

$$
T_{z} \text { is bounded on } L^{2}\left(\mathbb{R}^{4}\right) \text { when } \operatorname{Re}(z)=-\frac{3}{2}
$$

and, if $H=\left(\frac{1}{3}, \frac{1}{6}\right)$, that

$$
T_{z} \text { maps } L^{p}\left(\mathbb{R}^{4}\right) \text { into } L^{q}\left(\mathbb{R}^{4}\right) \text { if }\left(\frac{1}{p}, \frac{1}{q}\right) \in[G, H) \text { and } \operatorname{Re} z=-\frac{2}{3} .
$$

Analytic interpolation and the fact that $T_{-1}$ is a multiple of $T$ then show that $[G, B) \subseteq \mathcal{T}$ as claimed. by

To establish (1) we need to estimate the Fourier transform $\widehat{T}_{-\frac{3}{2}+i s}$ given formally

$$
\widehat{T}_{-\frac{3}{2}+i s}(y)=\frac{1}{\Gamma\left(\left(-\frac{1}{2}+i s\right) / 2\right)} \int_{0}^{1} \int_{-1}^{1} e^{-i y \cdot\left(\gamma(t)+u \gamma^{\prime \prime}(t)\right)}|u|^{-\frac{3}{2}+i s} d u d t .
$$

Because the integral

$$
\int_{-1}^{\infty}|u|^{-\frac{2}{3}} d u
$$

converges, it is enough to estimate the integral

$$
\int_{0}^{1} \int_{-\infty}^{\infty} e^{-i y \cdot\left(\gamma(t)+u \gamma^{\prime \prime}(t)\right)}|u|^{-\frac{3}{2}+i s} d u d t
$$

If $y=\left(y_{1}, y_{2}, y_{3}, y_{4}\right)$, write $p(t)=\sum_{j=1}^{4} y_{j} t^{j}$. Since the Fourier transform of the one-dimensional distribution $|u|^{-\frac{3}{2}+i s} d u$ is a multiple of $|x|^{\frac{1}{2}-i s} d x$, (3) is essentially

$$
\int_{0}^{1} e^{-i p(t)}\left|p^{\prime \prime}(t)\right|^{\frac{1}{2}-i s} d t
$$

A result of [O3] bounds this integral by $C(1+|s|)^{\frac{1}{2}}$ independently of $y$. Thus

$$
\left\|\widehat{T}_{-\frac{3}{2}+i s}\right\|_{\infty} \leq C(1+|s|)^{\frac{1}{2}},
$$

yielding (1).

To prove (2) we begin by defining

$$
\begin{aligned}
& S_{1} f(x)=\int_{0}^{\infty} \int_{\frac{1}{2}}^{1} f\left(x-\gamma(t)-u \gamma^{\prime \prime}(t)\right) d u d t \doteq \mu_{1} * f(x), \\
& S_{2} f(x)=\int_{0}^{\infty} \int_{-1}^{-\frac{1}{2}} f\left(x-\gamma(t)-u \gamma^{\prime \prime}(t)\right) d u d t \doteq \mu_{2} * f(x) .
\end{aligned}
$$

With $S=S_{1}+S_{2}$, our immediate goal is to establish that

$$
S \text { maps } L^{3}\left(\mathbb{R}^{4}\right) \text { into } L^{6}\left(\mathbb{R}^{4}\right) \text {. }
$$

We will do this by adapting an argument from [O2]. Now (4) follows from the two multilinear estimates

$$
\left|\int_{\mathbb{R}^{4}} \prod_{j=1}^{6} \mu_{\ell} * f_{j}(x) d x\right| \leq C \prod_{j=1}^{6}\left\|f_{j}\right\|_{3}, \quad \ell=1,2 .
$$


Utilizing Christ's remarkable observation about multilinear interpolation ([C1], pp. 227-228), (5) will follow from the estimates

$$
\left|\int_{\mathbb{R}^{4}} \prod_{j=1}^{6} \mu_{\ell} * f_{j}(x) d x\right| \leq C\left\|f_{1}\right\|_{1} \prod_{j=2}^{6}\left\|f_{j}\right\|_{5,1}, \quad \ell=1,2,
$$

where the symbol $\|\cdot\|_{p, r}$ stands for a Lorentz space norm on $\mathbb{R}^{4}$. It is enough to prove (6) with $f_{1}$ replaced by the point mass at the origin in $\mathbb{R}^{4}$ and so (6) reduces to the estimate

$$
\left\|S_{\ell} f\right\|_{L^{5}(\mu)} \leq C\|f\|_{5,1} .
$$

We will actually establish the dual of this estimate,

$$
\left\|S_{\ell}^{*} g\right\|_{\frac{5}{4}, \infty} \leq C\|g\|_{L^{\frac{5}{4}}\left(\mu_{\ell}\right)},
$$

for functions $g$ on the two-dimensional manifold that is the support of $\mu_{\ell}$. Let $\phi$ be the map of $\mathbb{R}^{4}$ into itself defined by

$$
\phi(t, u, s, v)=\gamma(t)+u \gamma^{\prime \prime}(t)-\gamma(s)-v \gamma^{\prime \prime}(s) .
$$

Computations show that

$$
\phi \text { is at most three to one on the set }\{t \neq s\}
$$

and that the absolute value $J$ of the Jacobian of $\phi$ is given by

$$
J(t, u, s, v)=24(s-t)^{2}\left|(s-t)^{2}-6(u+v)\right| .
$$

Let $m$ denote Lebesgue measure on $\mathbb{R}^{4}$. From (8) it follows that if $U \subseteq \mathbb{R}^{4}$ is open and if the measure $\lambda_{U}$ is defined on $\phi(U)$ by

$$
\int_{\phi(U)} h d \lambda_{U}=\int_{U} h \circ \phi(t, u, s, v) J(t, u, s, v) d(t, u, s, v),
$$

then

$$
m(E) \leq \lambda_{U}(E) \leq 3 m(E), \quad E \subseteq U .
$$

Let $Q_{1}=\left\{(s, v): 0<s<\infty, \frac{1}{2}<v<1\right\}, Q_{2}=\{(s, v): 0<s<\infty,-1<v<$ $\left.-\frac{1}{2}\right\}$. If $f$ is a function on $\mathbb{R}^{4}$ and $g$ is defined on the support of $\mu_{\ell}$, then we have, writing $g(s, v)$ for $g\left(\gamma(s)+v \gamma^{\prime \prime}(s)\right)$,

$$
\begin{aligned}
\int_{\mathbb{R}^{4}} f(x) S_{\ell}^{*} g(x) d x & =\int_{\mathbb{R}^{4}} S_{\ell} f(y) g(y) d \mu_{\ell}(y) \\
& =\int_{Q_{\ell} \times Q_{\ell}} f(\phi(t, u, s, v)) \frac{g(s, v)}{J(t, u, s, v)} J(t, u, s, v) d(t, u, s, v) .
\end{aligned}
$$

The last integral is bounded by the product of the two Lorentz norms

$$
\|f \circ \phi\|_{L^{5,1}(J d m)}, \quad\|g / J\|_{L^{\frac{5}{4}, \infty}(J d m)} .
$$

By (10) the first of these is bounded by $\|f\|_{5,1}$. We will establish (7) by observing that there is a constant $C$ such that, for any $\alpha>0$,

$$
\int_{\left(Q_{\ell} \times Q_{\ell}\right) \cap\{|g(x, v)|>\alpha J(t, u, s, v)\}} J(t, u, s, v) d(t, u, s, v) \leq \frac{C}{\alpha^{\frac{5}{4}}} \int_{Q_{\ell}}|g(s, v)|^{\frac{5}{4}} d(s, v) .
$$


This will follow from the inequality, uniform in $A>0$ and $(s, v) \in Q_{\ell}$,

$$
\int_{Q_{\ell} \cap\{J(t, u, s, v)<A\}} J(t, u, s, v) d(t, u) \leq C A^{\frac{5}{4}} .
$$

Taking account of (9) it is enough to observe that there is $C$ such that for $A>0$ and $6<|k|<12$ we have

$$
\int_{\left\{t^{2}\left|t^{2}-k\right|<A\right\}} t^{2}\left|t^{2}-k\right| d t \leq C A^{\frac{5}{4}}
$$

But this inequality is easily checked by considering separately the cases of large and small $A$. The first case follows from the fact that if $|t|$ is large, then $t^{2}\left|t^{2}-k\right| \sim t^{4}$, leading to (11). In the case of small $A, t^{2}\left|t^{2}-k\right|<A$ implies either $t^{2}\left|t^{2}-k\right| \sim C t^{2}$ or $t^{2}\left|t^{2}-k\right| \sim C \mid t \pm \sqrt{k}$, leading to estimates of $C A^{\frac{3}{2}}$ or $C A^{2}$ respectively for the left-hand side of (11). These complete the proof of (4).

Now define operators $V_{k}$ by

$$
V_{k} f(x)=\int_{0}^{1} \int_{2^{-k}<|u|<2^{-k+1}} f\left(x-\gamma(t)-u \gamma^{\prime \prime}(t)\right) d u d t .
$$

Let $\left\|V_{k}\right\|_{p q}$ denote the norm of $V_{k}$ from $L^{p}\left(\mathbb{R}^{4}\right)$ to $L^{q}\left(\mathbb{R}^{4}\right)$. Then, since

$$
T_{-\frac{3}{2}} \sim \sum_{k=1}^{\infty} 2^{\frac{2 k}{3}} V_{k},
$$

(2) will follow from the estimate

$$
\left\|V_{k}\right\|_{p q} \leq C 2^{-k(1-2 \alpha)}
$$

for $\left(\frac{1}{p}, \frac{1}{q}\right)$ on $[G, H)$ with $\frac{1}{p}-\frac{1}{q}=\alpha$ along with the fact that $\alpha<\frac{1}{6}$. Inequality (12) comes from interpolating the cases $\alpha=0$ (corresponding to $G=\left(\frac{1}{2}, \frac{1}{2}\right)$ ) and $\alpha=\frac{1}{6}$ (corresponding to $\left.H=\left(\frac{1}{3}, \frac{1}{6}\right)\right)$. The first of these two estimates is clear. The second is a consequence of (4) and a homogeneity argument:

For $x=\left(x_{1}, x_{2}, x_{3}, x_{4}\right) \in \mathbb{R}^{4}$ and $\lambda \in \mathbb{R}$, let $[\lambda] x$ be the nonisotropic dilate of $x$ given by $\left(\lambda x_{1}, \lambda^{2} x_{2}, \lambda^{3} x_{3}, \lambda^{4} x_{4}\right)$. For $\lambda>0$, let $V_{[\lambda]}$ be the dilate of $V$ given by

$$
V_{[\lambda]} f(x)=\int_{0}^{\infty} \int_{\frac{1}{2}<|u|<1} f\left(x-[\lambda]\left(\gamma(t)+u \gamma^{\prime \prime}(t)\right)\right) d u d t .
$$

Then it is easy to see that

$$
\left\|V_{[\lambda]}\right\|_{p q}=\lambda^{-\left(\frac{10}{p}-\frac{10}{q}\right)}\|S\|_{p q}
$$

and that

$$
V_{[\lambda]} f(x)=\lambda^{-3} \int_{0}^{\infty} \int_{\frac{\lambda^{2}}{2}<|u|<\lambda^{2}} f\left(x-\gamma(t)-u \gamma^{\prime \prime}(t)\right) d u d t .
$$

Taking $\lambda=2^{-\frac{k}{2}}$ and combining these two observations with (4) gives (12) for $\alpha=\frac{1}{6}$ (and $\left.\left(\frac{1}{p}, \frac{1}{q}\right)=H=\left(\frac{1}{3}, \frac{1}{6}\right)\right)$.

\section{ADDED IN PROOF}

The recent paper [GSW] uses a modification of the method of this paper to show that $T$ is of restricted weak type at the points $B$ and $E$. And the more recent paper [C2] uses an entirely different (geometric) method to prove the $n$-dimensional analogue of this last result. 


\section{REFERENCES}

[C1] M. Christ, On the restriction of the Fourier transform to curves: endpoint results and the degenerate case, Trans. Amer. Math. Soc. 287 (1985), 223-238. MR 87b:42018

[C2] M. Christ, Convolution, Curvature and Combinatorics, a Case Study, preprint.

[GSW] A. Greenleaf, A. Seeger, and S. Wainger, On x-ray transforms for rigid line complexes and integrals over curves in $\mathbb{R}^{4}$, preprint.

[O1] D. Oberlin, A convolution estimate for a measure on a curve in $\mathbb{R}^{4}$, Proc. Amer. Math. Soc. 125 (1997), 1355-1361. MR 97g:42009

[O2] D. Oberlin, Multilinear proofs for two theorems on circular averages, Colloq. Math. 63 (1992), 187-190. MR 93m:42005

[O3] D. Oberlin, Oscillatory integrals with polynomial phase, Math. Scand. 69 (1991), 45-56.

Department of Mathematics, Florida State University, Tallahassee, Florida 32306 4510 